\title{
openheart Exercise stress echocardiography: a great tool that can be adapted to the clinical question?
}

\author{
Erwan Donal (D) , 'asileios Panis, ${ }^{1}$ Wojciech Kosmala ${ }^{2}$
}

To cite: Donal E, Panis V, Kosmala W. Exercise stress echocardiography: a great tool that can be adapted to the clinical question?. Open Heart 2021;8:e001641. doi:10.1136/ openhrt-2021-001641

Accepted 13 April 2021

\section{SLinked}

- http://dx.doi.org/10.1136/ openhrt-2021-001583

Check for updates

(C) Author(s) (or their employer(s)) 2021. Re-use permitted under CC BY-NC. No commercial re-use. See rights and permissions. Published by BMJ.

${ }^{1}$ Cardiology, CHU de Rennes Inserm, LTSI-UMR 1099,

Rennes, Bretagne, France

${ }^{2}$ Cardiology, Wroclaw Medical University, Wroclaw, Dolnoslaskie, Poland

Correspondence to Dr Erwan Donal; erwan.donal@ chu-rennes.fr
In recent years, there has been an increasing use of stress echocardiography in the assessment of non-ischaemic cardiac disease. Exercise stress echocardiography (ESE) provides a unique ability for simultaneous assessment of both cardiac performance and exerciserelated non-invasive haemodynamic changes, which can help guide treatment and inform about the prognosis of patients. ${ }^{1}$

The recommendations of European Association for Cardiovascular Imaging and American Society of Echocardiography have tried to standardise the use of ESE for nonischaemic purposes. ${ }^{1}$

In an echocardiography laboratory, we do not only detect wall motion abnormalities resulting from coronary artery stenosis, but also micro-vessels' disease, left ventricular systolic and diastolic dysfunction, left ventricular outflow tract obstruction, heart valve diseases and their consequences for the pulmonary circulation and the right ventricle. ${ }^{1}$

ESE has been proposed as a diagnostic tool in several pathologies, such as aortic stenosis, mitral regurgitation (MR) and hypertrophic cardiomyopathy. ${ }^{23}$ Diastolic stress test is advocated by recommendations and consensus papers, and its clinical use is increasing. ${ }^{45} \mathrm{In}$ the daily clinical practice, it is very simple and informative. In contrast to some other indications, diastolic ESE is based on standardised protocols permitting uniform assessment and ensuring good reproducibility. ${ }^{45}$

The two basic modalities for ESE are a treadmill and bicycle, preferably semisupine on a tilt table, which provides optimal imaging information on cardiac responses to stress (figure 1).

In MR, there is evidence that ESE can assist in the diagnostic process, revealing the dynamic nature of valve incompetence. Exercise-induced increase in the degree of regurgitation and/or increase in systolic pulmonary artery pressure (SPAP) are used as markers of the clinical significance of MR despite its non-severe status at rest. In patients with severe MR on resting imaging, exercise assessment should focus on SPAP and left ventricle (LV) systolic reserve, which might provide prognostic implications. ${ }^{6}$

In this issue of Open Heart, Kagiyama et al compared the physiological effects and prognostic value of another stress modalityhandgrip with a semisupine bicycle $\mathrm{ESE}^{7}$ in patients with MR. In contrast to dynamic exercise associated with bicycling, handgrip provides isometric exercise with somewhat different impact on haemodynamics. The potential advantage of handgrip is the simplicity of application and feasibility for a wider proportion of patients, however, the clinical utility of this approach is poorly validated.

Exercise-induced functional MR may affect functional capacity and outcome. Therefore, a 'proper' exploration of dynamic changes in functional MR is clinically important. ${ }^{1}$ Kagiyama $e t$ al have shown that MR can increase during handgrip-ESE to the extent even larger than during bicycle ESE, however, increases in LV contractile state and SPAP are significantly smaller than those induced by dynamic exercise. Moreover, only data acquired during bicycle ESE-LV contractile reserve by global longitudinal strain proved to be of prognostic significance, which provides implications for patient management. Accordingly, the clinical value seems to be better for the 'classical' bicycle ESE. ${ }^{8}$

However, a semisupine bicycle ESE is sometimes challenging as it requires a dedicated equipment and cannot be performed in each patient due to, for example, orthopaedic problems. Therefore, alternative stress testing modalities are sought to circumvent the above limitations.

Is there a place for handgrip in these competitions? In view of the similar or even larger effect on MR volume in comparison 


\section{standardized stress test}

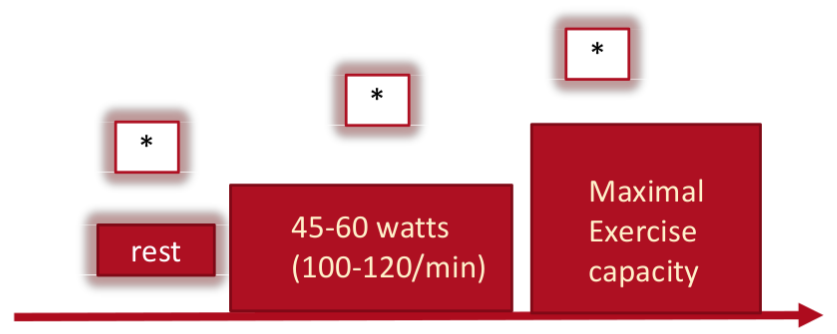

Complete Echo*

Left ventricular strain + Left Atrial volumetstrain, e', E/e', Vmax Tricuspid regurgitation,

cardiac output, 4-3- 2- apical views with and without color doppler + assessment of

regurgitations especially mitral regurgitation (dynamic functional mitral regurgitation)

Figure 1 Protocol for stress echocardiography in patients with suspected cardiac dyspnoea.

with bicycle ESE, handgrip might be considered as a first-choice diagnostic option in patients requiring stress testing to evaluate MR severity, especially in those unable to perform dynamic exercise. However, given the aforementioned differences in cardiac responses to both types of exercise demonstrated by Kagiyama, the definite conclusions should be made cautiously.

An important inference from the study by Kagiyama is that isometric and dynamic exercise do not provide equivalent clinical information in MR. A semisupine bicycle ESE better addresses SPAP and LV systolic reserve, and, consequently, outperforms handgrip ESE as a prognosticating tool.

The work of Kagiyama $e t$ al helps in advocating for a wider use of ESE in MR according to a more standardised approach as has been proposed in the respective position papers. The message from this study is important because ESE could certainly reach a better level of recommendation in the guidelines dedicated to MR. For the time being, we are at the beginning of the road and more investigations are needed to support and extend the findings of Kagiyama et al.

\section{Twitter Erwan Donal @DonalErwan}

Contributors All the authors contributed significantly.

Funding The authors have not declared a specific grant for this research from any funding agency in the public, commercial or not-for-profit sectors.

Competing interests None declared.

Patient consent for publication Not required.

Provenance and peer review Commissioned; internally peer reviewed.

Data availability statement No additional data are available.

Open access This is an open access article distributed in accordance with the Creative Commons Attribution Non Commercial (CC BY-NC 4.0) license, which permits others to distribute, remix, adapt, build upon this work non-commercially, and license their derivative works on different terms, provided the original work is properly cited, appropriate credit is given, any changes made indicated, and the use is non-commercial. See: http://creativecommons.org/licenses/by-nc/4.0/.

ORCID iD

Erwan Donal http://orcid.org/0000-0003-2677-3389

\section{REFERENCES}

1 Lancellotti P, Pellikka PA, Budts W, et al. The clinical use of stress echocardiography in non-ischaemic heart disease: recommendations from the European association of cardiovascular imaging and the American Society of echocardiography. Eur Heart J Cardiovasc Imaging 2016;17:1191-229.

2 Ommen SR, Mital S, Burke MA, et al. 2020 AHA/ACC guideline for the diagnosis and treatment of patients with hypertrophic cardiomyopathy: a report of the American College of Cardiology/ American heart association joint Committee on clinical practice guidelines. J Am Coll Cardiol 2020;76:e159-240.

3 Elliott P, Andersson B, Arbustini E, et al. Classification of the cardiomyopathies: a position statement from the European Society of cardiology Working group on myocardial and pericardial diseases. Eur Heart J 2008;29:270-6.

4 Pieske B, Tschöpe C, de Boer RA, et al. How to diagnose heart failure with preserved ejection fraction: the HFA-PEFF diagnostic algorithm: a consensus recommendation from the heart failure association (HFA) of the European Society of cardiology (ESC). Eur Heart $J$ 2019;40:3297-317.

5 Ponikowski P, Voors AA, Anker SD, et al. 2016 ESC Guidelines for the diagnosis and treatment of acute and chronic heart failure: The Task Force for the diagnosis and treatment of acute and chronic heart failure of the European Society of Cardiology (ESC)Developed with the special contribution of the Heart Failure Association (HFA) of the ESC. Eur Heart J 2016;37:2129-200.

6 Magne J, Donal E, Mahjoub H, et al. Impact of exercise pulmonary hypertension on postoperative outcome in primary mitral regurgitation. Heart 2015;101:391-6.

7 Kagiyama NTM, Yuri T, Aritaka S. Physiologic and prognostic differences between types of exercise stress echocardiography for functional mitral regurgitation. Open Heart 2021.

8 Alachkar MN, Kirschfink A, Grebe J, et al. Dynamic handgrip exercise for the evaluation of mitral valve regurgitation: an echocardiographic study to identify exertion induced severe mitral regurgitation. Int $J$ Cardiovasc Imaging 2021;37:891-902. 